\title{
Reoccurrence of Bleeding of a Chronic Subdural Haematoma Following a Fall
}

\author{
Rafael García Carretero* \\ Internal Medicine Department, Mostoles University Hospital, Rio Jucar, 28935, Mostoles (Madrid), Spain
}

\begin{abstract}
The case of a 60-year-old patient who presented with an acute-on-chronic subdural haematoma is reported. Chronic haematoma usually remains asymptomatic, and this is considered to be an unusual course of events. Trivial or minor injury may cause the cortical bridge veins and fragile vessels in the former haematoma to rupture with concomitant reoccurrence of bleeding. Old age, repeated traumatic brain injuries, brain atrophy, antiplatelet agents and oral anticoagulants such as warfarin are considered to be the underlying conditions to cause the reoccurrence of bleeding. However, our patient did not have any of those conditions.
\end{abstract}

Keywords: chronic subdural hematoma, reoccurrence of bleeding, acute-on-chronic subdural hematoma

Received: 9 June 2017 / Accepted:12 July 2017

\section{INTRODUCTION}

Although uncommon, acute-on-chronic subdural haematoma (ACSDH) is not rare. Lee et al. (2004) found that $8 \%$ of chronic subdural haematomas $(\mathrm{CSDH})$ were actually ACSDHs, $[1,2]$ although there are few cases reported in medical literature. Chronic haematomas develop by the pooling of blood caused by tears in the bridging vessels in the subdural space. Old age, repeated trauma and brain atrophy are the main risk factors. Unlike acute subdural haematomas, which may be life-threatening, chronic haemorrhages have a better prognosis. Some chronic subdural haematomas remain asymptomatic, but a mild injury or brain trauma may lead to the subdural reoccurrence of bleeding.

\section{CASE REPORT}

A 60-year-old man was admitted to hospital following a mild traumatic head injury. He was lethargic and stuporous.

His wife reported that six months before, he had accidentally fallen and hit his head and had been admitted to hospital.

At that visit, he had no neurological symptoms, except for headache and nausea. No lower or upper limbs paresis were present. A brain CT was performed, and the definitive diagnosis was an acute left frontoparietal subdural haematoma. Given it was small in size and the lack of severe symptoms after 48 hours of observation, the supervising clinicians decided to manage the case with careful monitoring over time. In accordance with this policy, a second brain CT scan was carried out several weeks later, which did not show any changes compared to the previous CT scan (Figure 1). This, together with the fact that the patient had been asymptomatic since the initial admission to hospital, led to a diagnosis of chronic subdural haematoma.

At the time of his second admission to hospital, he was assessed in the Emergency Medicine Department. His relatives claimed that the trauma to his head had been mild, explaining that he had taken a nap and had rolled over and fallen off the couch. After a few minutes, he showed decreased alertness and was unable to follow simple commands or speak.

On physical examination, his pupils were normal, but right hemiparesis was present. The patient was not on any drug treatment, nor was he taking any vitamin $\mathrm{K}$ antagonists or antiplatelet agents.

The patient then underwent a brain CT scan, which showed acute reoccurrence of bleeding of the previous chronic subdural haematoma (Figure 1). The patient was referred to the Neurosurgery Department to undergo surgical drainage, after which he fully recovered.

\footnotetext{
* Correspondence to: Rafael García Carretero, Internal Medicine Department, Mostoles University Hospital, Rio Jucar, 28935, Mostoles (Madrid), Spain. E-mail: rgcarretero@salud.madrid.org
} 

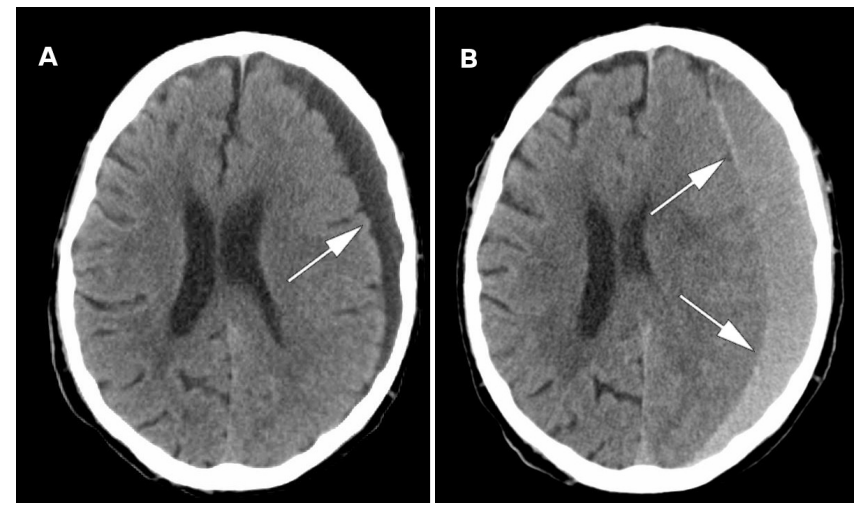

Fig. 1. A: Axial slide of a brain CT scan, showing a chronic subdural haematoma (white arrow), $13 \mathrm{~mm}$ thick and mild midline shift to the right $(8 \mathrm{~mm})$. B: The current CT scan showed an acute crescent-shaped haematoma, 21 $\mathrm{mm}$ thick, causing mass-effect with raised intracranial pressure and midline shift to the right (12 $\mathrm{mm})$. Chronic haematoma is hypodense, whereas acute (or acute-onchronic) haematoma is hyperdense.

\section{DISCUSSION}

Chronic haematomas are a mixture of liquefied blood and a semisolid clot pooling between the dura mater and the arachnoid mater $[1,3]$. Although the pathophysiology of ACSDH is complex and incompletely understood, it appears that rotational forces accompanying movement of the skull exert tensile strain and rupture of bridging veins leading to acute haemorrhaging into the subdural space [4]. When acute onset occurs, subdural haematomas can be life-threatening though some chronic haematomas remain asymptomatic and have a good prognosis. Brain atrophy after a traumatic brain injury is considered to be one of the underlying conditions that leads to the development of chronic haematomas. Elderly and alcoholic patients, with a history of repeated trauma or receiving treatment such as antiplatelet agents or warfarin, are more likely to develop this kind of haematoma. A chronic subdural haematoma leads to an excessive local activation of coagulation and fibrinolytic events, and a new onset haematoma would not necessarily form a solid clot [2].

Nevertheless, acute-on-chronic haematomas are not very common, representing about $8 \%$ of chronic subdural haematomas $[5,6]$. Removal of the lesion may re- lieve the pressure on the brain and resolve the midline shift.

\section{CONCLUSIONS}

Underlying conditions leading to a chronic subdural haematoma are old age, repeated trauma and brain atrophy. However, repetitive trauma can cause a reoccurrence of bleeding of a chronic haematoma even after a mild traumatic brain injury.

This case highlights the fact that acute bleeding over a chronic subdural haematoma may be identified by the onset of acute symptoms.

\section{CONFLICTS OF INTEREST}

The author has no competing interests in the manuscript.

\section{ACKNOWLEDGEMENTS}

I would like to thank Jacqueline Lamb for her great support, suggestions and encouragement in the making of this manuscript.

\section{REFERENCES}

1. Lee KS. Natural history of chronic subdural haematoma. Brain Inj. 2004;18:351-8.

2. Lee KS, Shim JJ, Yoon SM, Doh JW, Yun IG, Bae HG. Acute-onChronic Subdural Hematoma: Not Uncommon Events. J Korean Neurosurg Soc. 2011;50:512-6.

3. Kloss BT, Lagace RE. Acute-on-chronic subdural hematoma. Int J of Emerg Med. 2010;3:511-2.

4. Castellani RJ, Mojica-Sanchez G, Schwartzbauer G, Hersh DS. Symptomatic Acute-on-Chronic Subdural Hematoma: A Clinicopathological Study. American J Forensic Med Pathol. 2017;38:126-30.

5. Miele VJ, Carson L, Carr A, Bailes JE. Acute on chronic subdural hematoma in a female boxer: a case report. Med Sci Sports Exerc. 2004;36:1852-5.

6. Park IB, Moon SY, Kim YY, Kwon YE, Lee JH. Acute-on-chronic subdural hematoma by spinal anesthesia in a patient with undiagnosed chronic subdural hematoma: A case report. Korean J Anesthesiol. 2011;61:75-8. 